\title{
Incidence of Acute Cardiovascular Event After Acute Exacerbation of COPD
}

\author{
Tadahiro Goto, MD, $\mathrm{MPH}^{7}$, Yuichi J. Shimada, $\mathrm{MD}, \mathrm{MPH}^{2}$, Mohammad Kamal Faridi, $\mathrm{MPH}^{7}$, \\ Carlos A. Camargo Jr, MD, $\mathrm{DrPH}^{7}$, and Kohei Hasegawa, MD, MPH
}

'Department of Emergency Medicine, Massachusetts General Hospital, Boston, MA, USA; ${ }^{2}$ Cardiology Division, Department of Medicine, Massachusetts General Hospital, Boston, MA, USA.

BACKGROUND: There is a lack of comprehensive view of the association between acute exacerbation of COPD (AECOPD) and the risk of acute cardiovascular events.

OBJECTIVE: To determine the association of AECOPD with 30-day and 1-year incidences of acute cardiovascular event.

DESIGN: Self-controlled case series analysis using population-based datasets from three US states from 2005 through 2011.

PARTICIPANTS: Patients aged $\geq 40$ years with AECOPD. MAIN MEASURES: The primary outcome was a composite of an ED visit or hospitalization for acute cardiovascular events, including acute myocardial infarction, heart failure, atrial fibrillation, pulmonary embolism, and stroke. We compared the incidence of each patient's acute cardiovascular event during the first 30-day period before the index AECOPD (30-day reference period) in comparison with that during the 30-day period after the index AECOPD. Likewise, with the 1-year period before the index AECOPD as reference, we also estimated incidence rate ratios (IRRs) for each patient's outcomes during 1year period after the index AECOPD.

KEY RESULTS: Overall, there were 362,867 patients with an ED visit or hospitalization for AECOPD. Compared with the 30-day reference period, the incidence of acute cardiovascular event in the 30-day period after the AECOPD was significantly higher (IRR, 1.34; 95\%CI, 1.30-1.39; $P<0.001)$. Likewise, compared with the 1-year reference period, the incidence during the 1-year period after the AECOPD was also higher (IRR, 1.20; 95\%CI, 1.18-1.22; $P<0.001)$. For each of acute cardiovascular conditions, the associations remained significant (all $P<0.05$ ).

CONCLUSIONS: AECOPD was associated with increased 30-day and 1-year incidences of acute cardiovascular event.

KEY WORDS: acute exacerbation of COPD; COPD; cardiovascular diseases.

$J$ Gen Intern Med 33(9):1461-8

DOI: $10.1007 / \mathrm{s} 11606-018-4518-3$

() Society of General Internal Medicine 2018

Electronic supplementary material The online version of this article (https://doiorg/10.1007/s11606-018-4518-3) contains supplementary material, which is available to authorized users.

Received September 15, 2017

Revised April 12, 2018

Accepted May 24, 2018

Published online June 8, 2018

\section{INTRODUCTION}

Chronic obstructive pulmonary disease (COPD) is a major public health problem worldwide. ${ }^{1}$ The estimated prevalence of COPD is $12 \%$ globally, affecting nearly 400 million individuals in $2010 .^{2}$ The literature has reported that COPD is associated with a high prevalence of concomitant cardiovascular diseases, such as ischemic heart disease, heart failure $(\mathrm{HF})$, atrial fibrillation (AF), pulmonary embolism (PE), and stroke. $^{3,4}$ These diseases are the commonest comorbidities underlying causes of death in patients with $\mathrm{COPD} .{ }^{5-7}$ In this context, the American Thoracic Society and the European Respiratory Society have encouraged studies to examine relationships between COPD and comorbidities. ${ }^{8}$

Acute exacerbation of COPD (AECOPD) is an important adverse event during the clinical course of $\mathrm{COPD}^{9}$ accounting for 700,000 hospitalizations in the USA each year. ${ }^{10} \mathrm{~A}$ few community-based studies have reported that AECOPD may contribute to short-term risk of acute cardiovascular events (acute myocardial infarction $[\mathrm{AMI}]^{11}$ and ischemic stroke ${ }^{11,12}$ ). Furthermore, there are plausible biological mechanisms linking AECOPD to cardiovascular events, e.g., AECOPD-related increased inflammation and hypoxia lead to endothelium dysfunction, arterial and venous thromboembolic events, right heart strain, and cardiac remodeling. ${ }^{13-15}$ These studies suggest that AECOPD is associated with the risk of developing acute cardiovascular events. However, there is a lack of comprehensive view of the association between AECOPD and the risk of acute cardiovascular events including long-term risks. Better understanding of the risks of acute cardiovascular events after AECOPD would present an opportunity to implement preventive measures on this population at high risk.

To address these knowledge gaps, we analyzed large, population-based databases from three US states, over several years, to examine the association of AECOPD with short- and long-term incidences of acute cardiovascular events.

\section{MATERIALS AND METHODS}

\section{Study Design and Setting}

This is a self-controlled case series study using populationbased data from the State Emergency Department Database 
(SEDD) and State Inpatient Database (SID) of three US states (California, Florida, and Nebraska) from January 1, 2005 through December 31, 2011. This study design relies on intraperson comparisons in a population of subjects with both the exposure and outcome of interest. ${ }^{16}$ This design enables each subject to serve as his/her own control, and therefore, no separate control subjects are needed. Additionally, it has advantages that both measured and unmeasured timeinvariant confounders are implicitly eliminated. ${ }^{17}$ The SEDD and SID are a component of the Healthcare Cost and Utilization Project (HCUP) sponsored by the Agency for Healthcare and Research Quality. The HCUP includes the largest collection of longitudinal hospital care data in the USA with allpayer, encounter-level information. The SEDD captures all treat-and-release ED visits from short-term, acute-care, nonfederal hospitals in participating states. The SID captures all inpatient discharges from short-term, acute-care, non-federal, general, and other specialty hospitals. Additional details of the SEDD and SID may be found elsewhere. ${ }^{18}$ The study period was chosen based on the availability of databases across these three US states. The institutional review board of Massachusetts General Hospital approved this study.

\section{Study Population}

We identified all ED visits and hospitalizations made by patients aged $\geq 40$ years ${ }^{19}$ with a primary discharge diagnosis of COPD (ICD-9-CM diagnosis codes: 491.21, 491.22, 491.8, $491.9,492.8,493.20,493.21,493.22$, and 496) or those with a primary diagnosis of respiratory failure (ICD-9-CM diagnosis codes: 518.81, 518.82, 518.84, and 799.1) and a secondary diagnosis of COPD (see earlier codes). ${ }^{20}$ The use of these ICD-9-CM diagnosis codes to identify AECOPD had a high specificity and a positive predictive value (both $>90 \%$ ). ${ }^{21,22}$ To accommodate analyses of the 1-year period before the index ED visit or hospitalization for AECOPD (index AECOPD) and 1-year period after the index AECOPD, we included patients with an ED visit or hospitalization for AECOPD between January 1, 2006 and December 31, 2010. Additionally, to isolate the effect of the exposure (an ED visit or hospitalization for AECOPD) on the outcomes (acute cardiovascular events) within individuals using the selfcontrolled case-series design, we included only patients with one ED visit or hospitalization for AECOPD during 1-year periods before and after the index AECOPD. We excluded ED visits and hospitalizations by patients who left the hospital against medical advice, died in-hospital during their ED visit or hospitalization, transferred to another acute-care facility, and were out-of-state residents.

\section{Outcome Measures}

The primary outcome measure was a composite of an ED visit or non-elective hospitalization for acute cardiovascular events, including AMI (ICD-9-CM code 410.x1), ${ }^{23} \mathrm{HF}$ (ICD-9-CM codes 402.01, 402.11, 402.91, 404.01, 404.03, 404.11,
404.13, 404.91, 404.93, and 428), ${ }^{24,25} \mathrm{AF}$ (code 427.31), ${ }^{26}$ PE (code 415.1x), ${ }^{27}$ and stroke (codes 433.x1, 434.x1, and 436 for ischemic stroke; codes 430 and 431 for hemorrhagic stroke). ${ }^{28}$ For the secondary outcome measures, we examined ED visits and hospitalization for each of six acute cardiovascular conditions separately. The use of ICD-9-CM codes to identify these outcome measures was validated in the literature (e.g., positive predictive value of $97 \%$ for heart failure, ${ }^{29} 96 \%$ for coronary artery diseases, ${ }^{29}$ and $94 \%$ for ischemic stroke ${ }^{30}$ ).

\section{Statistical Analysis}

First, we described the baseline characteristics of patients who met the inclusion criteria. Next, we compared the incidence of each patient's acute cardiovascular event (i.e., the outcomes as count variable) during the first 30-day period before the index AECOPD (30-day reference period) in comparison with that during the 30-day period after the index AECOPD. We computed incidence rate ratios (IRRs) by fitting conditional Poisson regression models with the generalized estimating equations to account for the correlation over time. Likewise, with the 1-year period before the index AECOPD as reference, we also estimated IRRs for each patient's outcomes during 1-year period after the index AECOPD.

We also performed a series of sensitivity analyses to examine the consistency of inferences. First, we modeled the outcomes as binary variables by using conditional logistic regression models to compute the odds ratio (OR) for each patient's risk of outcome event during the study periods. Second, we repeated the primary analysis (i.e., conditional Poisson regression) with stratification by age category (40-64 years and $\geq$ 65 years) and sex. Third, we examined the exposure of interest-ED visit (with no resulting hospitalization) and hospitalization for AECOPD - separately. Fourth, to address the potential effect of loss to follow-up (e.g., out-of-hospital deaths and emigration from the three study states), we conducted a subgroup analysis limited to the patients who had any ED visit or hospitalization (regardless of reason) after 1 year or more from the index AECOPD. This subgroup analysis ensured that all patients were alive in the state until at least 12 months after the index AECOPD. Fifth, to address the potential association between advanced age and natural progression of cardiovascular risks, we analyzed the data using pre-AECOPD days 271 to 360 as the reference period and compared the incident rate of outcomes in post-AECOPD days 1 to 90 , days 91 to 180 , days 181 to 270 , and days 271 to 360 . Sixth, we analyzed data with a consideration of the issue of exposure causing delaying outcome. In this sensitivity analysis, we compared the incidence of cardiovascular events during 7-37 days before AECOPD and those during 30 days after AECOPD. Seventh, we repeated the analysis with stratification by hospital state on the primary outcome. Eighth, we conducted a subgroup analysis limited to patients with the primary diagnosis of COPD. Ninth, we have repeated the primary analysis including patients who had $\geq 1$ ED visit or hospitalization for AECOPD during 1-year periods before and after the index AECOPD (we used the first ED visit or hospitalization for AECOPD when a 
patient had multiple AECOPD events). Tenth, to address the potential suboptimal validity of outcome case definitions, we conducted a sensitivity analysis using all-cause hospitalization as an outcome measure. Lastly, we have analyzed data with stratification by hospital length-of-stay $(0-1,2-5$, and $>$ 5 days) according to a previous study on the readmission risk after hospitalization for AECOPD. ${ }^{31}$ All analyses were performed using STATA 14.1 (StataCorp; College Station, TX). All $P$ values were two-tailed, with $P<0.05$ considered statistically significant.

\section{Patient Involvement}

No patients were involved in setting the research question or the outcome measures, nor were they involved in developing plans for design or implementation of the study. No patients were asked to advise on interpretation or writing up of results. There are no plans to disseminate the results of the research to study participants or the relevant patient community.

\section{RESULTS}

From January 1, 2006 through December 31, 2010, there were 627,878 patients with $\geq 1$ hospitalization for AECOPD during 2005-2011 in three US states, corresponding 1,254,792 hospitalizations for AECOPD. Of these, we excluded 201,757 patients with hospitalization for AECOPD in 2005 or 2011, 64,254 patients who met the any of exclusion criteria (including 30,289 patients with multiple AECOPD episodes during 1 -year periods before and after the index AECOPD), and remaining 362,867 patients were eligible for the primary analysis. Baseline characteristics of the study population are summarized in Table 1. Overall, the median age was 70 years (IQR, 59-79 years), and 57\% of patients were women. Most of patients were non-Hispanic white, Medicare beneficiaries, and living in urban area.

Figure 1 describes the incidence of acute cardiovascular events (ED visits or hospitalizations) within 30 days before and after the index AECOPD, and the corresponding IRRs. Compared with the 30-day reference period, significantly more acute cardiovascular events occurred during the 30-day period after the index AECOPD (7010 vs. 9414 acute cardiovascular events), with an IRR of 1.34 (95\% CI, 1.30-1.39; $P<0.001)$. In the analysis of secondary outcomes, this association remained significant across the six cardiovascular conditions (all $P<0.05$ ).

Figure 2 describes the incidence of acute cardiovascular events within 1 year before and after the index AECOPD, and the corresponding IRRs. Similar to the results using the 1-month periods, compared with the 1-year reference period, significantly more acute cardiovascular events occurred during the 1-year period after the index AECOPD (28,629 vs. 34,399 acute cardiovascular events) with an IRR of 1.20 (95\%CI, $1.18-1.22 ; P<0.001)$. For each of acute cardiovascular conditions, this significant association persisted (all $P<0.001$ ).
Table 1 Baseline Characteristics of Patients with Acute Exacerbation of COPD Who Are at Risk for Acute Cardiovascular Event

\begin{tabular}{ll}
\hline \hline Characteristics & $\mathbf{n = 3 6 2 , 8 6 7}$ \\
\hline Age (year), median (IQR) & $70(59-79)$ \\
Women, $n$ (\%) & $207,668(57)$ \\
Race/ethnicity, $n(\%)$ & $266,691(71)$ \\
Non-Hispanic white & $33,284(9)$ \\
Non-Hispanic black & $37,283(10)$ \\
Hispanics & $9629(3)$ \\
Asian or Pacific Islander & $360(1)$ \\
Native American & $5815(2)$ \\
Other & $21,413(6)$ \\
Missing & \\
Payment source, $n(\%)$ & $241,181(64)$ \\
Medicare & $38,946(10)$ \\
Medicaid & $56,267(15)$ \\
Private insurance & $23,243(6)$ \\
Self-pay & $3508(1)$ \\
Other & $11,330(3)$ \\
Missing & \\
Quartiles for median household income, $n(\%)$ & $118,259(32)$ \\
1 (lowest) & $101,592(27)$ \\
2 & $86,438(23)$ \\
3 & $59,296(16)$ \\
4 (highest) & $8890(2)$ \\
Missing & \\
Patient residence, $n(\%)$ & $337,013(90)$ \\
Urban & $35,456(9)$ \\
Rural & $2006(1)$ \\
Missing &
\end{tabular}

$C O P D$, chronic obstructive pulmonary disease; $I Q R$, interquartile range *Percentages may not equal to 100 due to rounding

A series of sensitivity analyses demonstrated the robustness of these findings. With modeling the outcomes as binary variables, the findings did not change materially from the primary analyses (eFigures 1 and 2). In the stratification analysis by age, a significantly higher incidence of acute cardiovascular events in the 30-day and 1-year periods after the index AECOPD was observed in most strata (eFigures 3 and 4). Similarly, in the stratified analysis by sex, a higher incidence of acute cardiovascular events during the 30-day and 1-year periods after the index AECOPD was observed across most strata (Figs. 3 and 4). Furthermore, in the sensitivity analysis that examined ED visit and hospitalization for AECOPD separately, there was a similar temporal pattern of the incidence for acute cardiovascular events (eFigures 5 and 6). In the subgroup analysis that included only patients known to be alive until at least 1 year after the index AECOPD, even with its limited statistical power, we observed a higher incidence of primary outcome measure in the 30-day and 1-year periods after the index AECOPD (eFigures 7 and 8 ; both $P<0.001$ ). In the analysis using preAECOPD days 271 to 360 as the reference period, the rate increased significantly in the first 90-day period immediately after the AECOPD hospitalization (Supplemental Table 1). Then, in the subsequent 90 -day periods, the rate decreased gradually. With a consideration of the issue of exposure causing delaying outcome, the IRRs of outcome measures remained significant (all, $P<0.05$; Supplemental Table 2). The IRR of primary outcome (composite of cardiovascular events) remained significant $(P<0.001)$ and was relatively higher compared to the main findings, suggesting the potential 


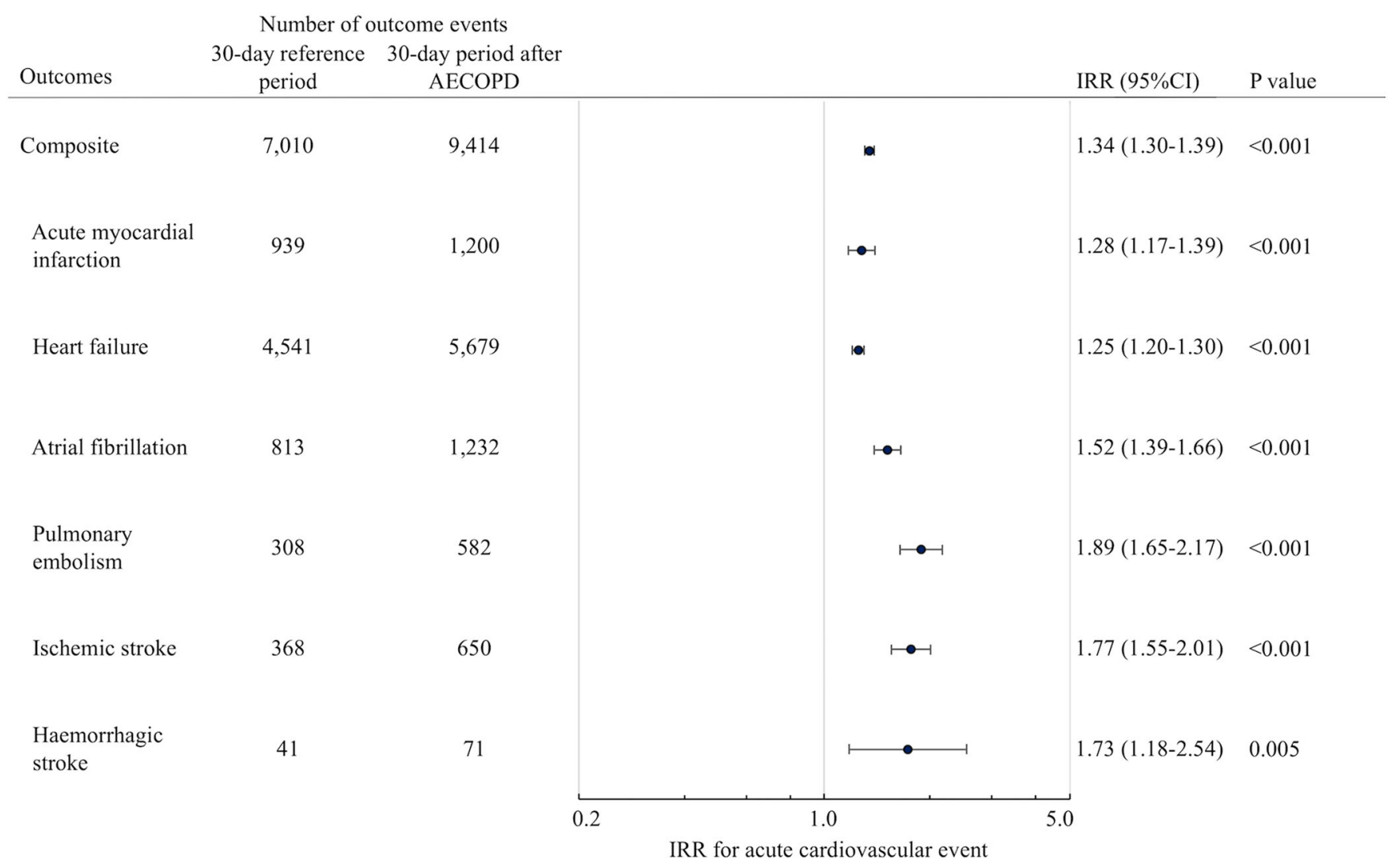

Figure 1 Incident rate ratios for acute cardiovascular event within 30 days after index acute exacerbation of COPD.

\begin{tabular}{|c|c|c|c|c|c|}
\hline Outcomes & $\begin{array}{l}\text { Number of } \\
\text { 1-year reference } \\
\text { period }\end{array}$ & $\begin{array}{l}\text { utcome events } \\
\text { 1-year period after } \\
\text { AECOPD }\end{array}$ & & $\operatorname{IRR}(95 \% \mathrm{CI})$ & $P$ value \\
\hline Composite & 28,629 & 34,399 & $\bullet$ & $1.20(1.18-1.22)$ & $<.001$ \\
\hline $\begin{array}{l}\text { Acute } \\
\text { myocardial } \\
\text { infarction }\end{array}$ & 3,879 & 4,253 & $\bullet$ & $1.10(1.05-1.15)$ & $<.001$ \\
\hline $\begin{array}{l}\text { Heart } \\
\text { failure }\end{array}$ & 18,283 & 21,880 & - & $1.20(1.17-1.22)$ & $<.001$ \\
\hline $\begin{array}{l}\text { Atrial } \\
\text { fibrillation }\end{array}$ & 3,275 & 3,902 & $\bullet$ & $1.19(1.14-1.25)$ & $<.001$ \\
\hline $\begin{array}{l}\text { Pulmonary } \\
\text { embolism }\end{array}$ & 1,181 & 1,504 & $1 \bullet-1$ & $1.27(1.18-1.37)$ & $<.001$ \\
\hline Ischemic stroke & 1,790 & 2,481 & |-1 & $1.39(1.30-1.47)$ & $<.001$ \\
\hline \multirow[t]{2}{*}{$\begin{array}{l}\text { Hemorrhagic } \\
\text { stroke }\end{array}$} & 221 & 379 & $\longmapsto$ & $1.71(1.45-2.02)$ & $<.001$ \\
\hline & & & 1.0 & 0 & \\
\hline
\end{tabular}

Figure 2 Incident rate ratios for acute cardiovascular event within 1 year after index acute exacerbation of COPD. 


\begin{tabular}{|c|c|c|c|c|c|c|}
\hline \multirow[b]{2}{*}{ Outcomes } & \multicolumn{3}{|c|}{ Number of outcome events } & & \multirow[b]{2}{*}{ IRR $(95 \% \mathrm{CI})$} & \multirow[b]{2}{*}{$\mathrm{P}$ value } \\
\hline & Sex & $\begin{array}{l}\text { 30-day reference } \\
\text { period }\end{array}$ & $\begin{array}{c}\text { 30-day period after } \\
\text { AECOPD }\end{array}$ & & & \\
\hline \multirow{2}{*}{ Composite } & Male & 3,389 & 4,353 & 1 & $1.28(1.23-1.34)$ & $<.001$ \\
\hline & Female & 3,607 & 5,039 & $1 \bullet$ & $1.40(1.34-1.46)$ & $<.001$ \\
\hline \multirow{2}{*}{$\begin{array}{l}\text { Acute } \\
\text { myocardial } \\
\text { infarction }\end{array}$} & Male & 463 & 592 & $\vdash \bullet-1$ & $1.28(1.13-1.44)$ & $<.001$ \\
\hline & Female & 475 & 605 & $\bullet \bullet$ & $1.27(1.13-1.44)$ & $<.001$ \\
\hline \multirow{2}{*}{ Heart failure } & Male & 2,219 & 2,736 & $\bullet$ & $1.23(1.17-1.30)$ & $<.001$ \\
\hline & Female & 2,311 & 2,930 & 101 & $1.27(1.20-1.34)$ & $<.001$ \\
\hline \multirow{2}{*}{ Atrial fibrillation } & Male & 372 & 516 & $\mapsto-1$ & $1.39(1.21-1.58)$ & $<.001$ \\
\hline & Female & 441 & 714 & $\mapsto \bullet$ & $1.62(1.44-1.82)$ & $<.001$ \\
\hline \multirow{2}{*}{$\begin{array}{l}\text { Pulmonary } \\
\text { embolism }\end{array}$} & Male & 143 & 236 & $\longmapsto$ & $1.65(1.34-2.03)$ & $<.001$ \\
\hline & Female & 164 & 345 & $\longmapsto$ & $2.01(1.75-2.53)$ & $<.001$ \\
\hline \multirow{2}{*}{ Ischemic stroke } & Male & 174 & 237 & $\longmapsto$ & $1.36(1.12-1.66)$ & .002 \\
\hline & Female & 193 & 410 & $\longmapsto$ & $2.12(1.79-2.52)$ & $<.001$ \\
\hline \multirow{3}{*}{$\begin{array}{l}\text { Hemorrhagic } \\
\text { stroke }\end{array}$} & Male & 18 & 36 & $\longmapsto$ & $2.00(1.14-3.52)$ & .02 \\
\hline & Female & 23 & 35 & $\longrightarrow$ & $1.52(0.90-2.58)$ & .12 \\
\hline & & & & lar outcome eve & 0 & \\
\hline
\end{tabular}

Figure 3 Incident rate ratios for acute cardiovascular event within 30 days after index acute exacerbation of COPD, by sex.

transient delay on the outcomes (Supplemental Table 3). Of 282,458 patients who were hospitalized with the primary diagnosis of COPD in the original cohort, the associations of AECOPD with the risk of subsequent cardiovascular diseases were similar to the main findings (Supplemental Table 4). In the sensitivity analysis of 393,156 patients who had multiple ED visits or/and hospitalizations for AECOPD during 1-year periods before and after the index AECOPD, as for the 30-day

\begin{tabular}{|c|c|c|c|c|c|c|}
\hline Outcomes & Sex & $\begin{array}{l}\text { Number of } \\
\text { 1-year reference } \\
\text { period }\end{array}$ & $\begin{array}{l}\text { utcome events } \\
\text { 1-year period after } \\
\text { AECOPD }\end{array}$ & & $\operatorname{IRR}(95 \% \mathrm{CI})$ & $P$ value \\
\hline \multirow{2}{*}{ Composite } & Male & 13,486 & 16,025 & 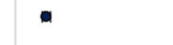 & $1.19(1.16-1.22)$ & $<.001$ \\
\hline & Female & 15,071 & 18,308 & - & $1.21(1.19-1.24)$ & $<.001$ \\
\hline \multirow{2}{*}{$\begin{array}{l}\text { Acute } \\
\text { myocardial } \\
\text { infarction }\end{array}$} & Male & 1,964 & 2,069 & 101 & $1.05(0.99-1.12)$ & .10 \\
\hline & Female & 1,907 & 2,171 & 101 & $1.14(1.07-1.21)$ & $<.001$ \\
\hline \multirow{2}{*}{ Heart failure } & Male & 8,686 & 10,446 & a & $1.20(1.17-1.24)$ & $<.001$ \\
\hline & Female & 9,549 & 11,397 & 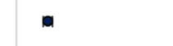 & $1.19(1.16-1.23)$ & $<.001$ \\
\hline \multirow{2}{*}{ Atrial fibrillation } & Male & 1,394 & 1,699 & 101 & $1.22(1.14-1.31)$ & $<.001$ \\
\hline & Female & 1,873 & 2,195 & $\bullet$ & $1.17(1.10-1.25)$ & $<.001$ \\
\hline \multirow{2}{*}{$\begin{array}{l}\text { Pulmonary } \\
\text { embolism }\end{array}$} & Male & 535 & 659 & $\mapsto-1$ & $1.23(1.10-1.38)$ & $<.001$ \\
\hline & Female & 642 & 844 & $\mapsto \bullet-1$ & $1.31(1.19-1.46)$ & $<.001$ \\
\hline \multirow{2}{*}{ Ischemic stroke } & Male & 813 & 1,006 & $1-1$ & $1.24(1.13-1.36)$ & $<.001$ \\
\hline & Female & 973 & 1,470 & $1 \bullet-1$ & $1.51(1.39-1.64)$ & $<.001$ \\
\hline \multirow{2}{*}{$\begin{array}{l}\text { Hemorrhagic } \\
\text { stroke }\end{array}$} & Male & 94 & 146 & $\longmapsto$ & $1.55(1.20-2.01)$ & .001 \\
\hline & Female & 127 & 231 & $\longmapsto$ & $1.82(1.46-2.26)$ & $<.001$ \\
\hline
\end{tabular}

Figure 4 Incident rate ratios for acute cardiovascular event within 1 year after index acute exacerbation of COPD, by sex. 
study period, the associations of interest did not change materially compared to the main findings (Supplemental Table 5). By contrast, as for the 1-year study period, the IRRs were relatively higher compared to the main findings. In the sensitivity analysis using all-cause hospitalization as an outcome measure, the magnitude of IRR for cardiovascular events was relatively higher compared to that for all-cause hospitalizations (Supplemental Table 6). Lastly, with stratification by hospital length-of-stay, the IRR for cardiovascular events in the 30-day period was relatively lower in patients with a longer length-ofstay compared to those with a shorter length-of-stay. As for the 1 -year study period, there were no apparent differences in IRR between the different hospital length-of-stay groups (Supplemental Table 7).

\section{DISCUSSION}

In this self-controlled case series study using the large population-based data of 362,867 patients with AECOPD, AECOPD was associated with increased 30-day and 1-year incidences of ED visits and hospitalizations for acute cardiovascular events. These associations remained significant across the six cardiovascular conditions (AMI, HF, AF, PE, ischemic stroke, and hemorrhagic stroke). Furthermore, the observed higher incidences of acute cardiovascular event persisted across several different analytic assumptions.

Few studies have reported the association between AECOPD and the risks of developing AMI and stroke. ${ }^{11,12}$ A communitybased study of 25,857 patients with COPD from England reported that AECOPD is associated with a 2.3-fold higher risk of AMI within 1 to 5 days after exacerbation (defined by prescription of both steroids and antibiotics) and a 1.3-fold higher risk of ischemic stroke 1 to 49 days after exacerbation. Similarly, another community-based study of 1556 patients with COPD from Belgium reported that the risk of stroke (composite of all types of stroke) during 7-week period after severe AECOPD was sixfold higher than that of stable disease. ${ }^{12}$ Our observations are consistent with these earlier findings, and we extend them by demonstrating both short- and long-term incidences of acute cardiovascular events more comprehensively in the population-based data with a sample size many times larger than any other prior studies on this topic.

We found that AECOPD is associated with a higher incidence of $\mathrm{HF}, \mathrm{AF}$, and PE during both short- and long-term periods. Although these three cardiovascular diseases are well-known comorbid conditions of $\mathrm{COPD},{ }^{7,32}$ only a few studies have investigated the association between AECOPD and the risk of developing acute events. Within the sparse literature, an analysis of 35 patients with COPD in Italy reported that impaired pulmonary function, hypercapnia, and higher pulmonary artery systolic pressure are predictors for new onset $\mathrm{AF}^{33} \mathrm{~A}$ case-control study from England $(n=3594)$ reported that the risk of venous thromboembolism did not differ between COPD patients with frequent exacerbations and those without. ${ }^{34}$ However, these previous studies were potentially limited by the small sample size, study design (e.g., cross-sectional study), and measurement of exposure (e.g., physiological values rather than AECOPD) ${ }^{34}$ By contrast, the validity of our findings is buttressed by the self-controlled case series design. This design enables each patient to serve as her/his own control, which minimizes the effect of unmeasured confounding that is inherent in traditional case-control and other observational study designs. ${ }^{17}$ Our study meets the requirements of the self-controlled case series design, in which we modeled a discrete and transient exposure and outcome that was defined by an acute event.

There are several plausible mechanisms linking AECOPD to acute cardiovascular events, particularly arterial and venous thromboembolic events. Most exacerbations of COPD are triggered by viral and/or bacterial airway infection ${ }^{13}$ which also leads to systemic inflammation with a rise in levels of plasma fibrinogen and C-reactive protein. ${ }^{35}$ The former is directly associated with thromboembolic events, while the latter upregulates other inflammatory mediators, leading to adhesion of leukocytes to arterial endothelium. ${ }^{36}$ Furthermore, arterial stiffness rises acutely during AECOPD particularly with airway infection. ${ }^{37}$ Acute infection in the setting of AECOPD leads to these changes that play a role in the pathobiology of acute cardiovascular events. ${ }^{14}$ Another potential mechanism is hypoxemia related to AECOPD. Hypoxemia increases pulmonary artery pressures, thereby leading to right heart strain and cardiac remodeling that contribute to AF pathobiology. ${ }^{15}$ Hypoxemia also leads to endothelial dysfunction, which predisposes to arterial and venous thromboembolic events. ${ }^{38}$ In addition to these biological mechanisms, non-biological iatrogenic factors (e.g., excessive use of $\beta_{2}$-agonist for AECOPD, cessation of $\beta$-blockers, ${ }^{39,40}$ and termination of aspirin in consideration of aspirinexacerbated respiratory diseases ${ }^{41}$ ) may also contribute, at least in part, to the increased risk of acute cardiovascular events in the long-term period after AECOPD. Indeed, $10-15 \%$ of patients discontinued $\beta$-blockers after AECOPD. ${ }^{39,40}$ While our findings underscore the importance of cardiovascular event risks in patients who are hospitalized for AECOPD, our study does not completely address the complexity of underlying mechanisms or specific prevention strategies. As recent studies have reported, further studies on specific prevention strategies should be facilitated, e.g., the use of aspirin may be beneficial for preventing not only cardiovascular diseases but also COPD progression. ${ }^{42,43}$

\section{Potential Limitations}

Our study has several potential limitations. First, because this analysis was based on the administrative data, misclassification of hospitalizations is possible. Nevertheless, the HCUP data are rigorously tested and widely used to estimate diagnoses and healthcare utilization. ${ }^{44}$ The $I C D-9-C M$ diagnosis codes that are used to identify AECOPD and acute cardiovascular events have high specificity and positive predictive values. ${ }^{21-23,28,29}$ 
Furthermore, potential misclassifications should not vary substantially between study periods, and therefore, any potential misclassifications are unlikely to have affected our inferences substantially. Second, the administrative datasets (i.e., SEDD and SID) do not include several helpful clinical variables, such as chronic severity of COPD and inpatient/outpatient management that are potentially associated with the risk of acute cardiovascular event. Nevertheless, our findings using the population-based data reflect the impact of AECOPD (including that of AECOPD management) on the risk of acute cardiovascular events in the natural setting of a real population and current clinical practice. Our findings should facilitate further investigation into the underlying mechanisms of observed associations. Third, due to the lack of out-of-hospital mortality data in the SEDD and SID, we were unable to directly account for the competing risk of mortality. However, this competing risk would have biased our inferences toward the null. Moreover, the subgroup analysis that included only patients known to be alive until at least 1 year after the index AECOPD still demonstrated a higher incidence of acute cardiovascular events after AECOPD, while this identification method of survival might have missed some healthier patients who survived but did not have any ED visits or hospitalizations. Finally, because the study population was comprised of patients with an ED visit or hospitalized for AECOPD, our inferences may not be generalizable to patients with mild AECOPD. Nevertheless, our study population has high morbidity and contributes to a substantial public health burden. ${ }^{10}$ Therefore, our findings are relevant to large numbers of high-risk and costly patients with COPD.

\section{CONCLUSIONS}

In this self-controlled case series study using large, populationbased datasets of patients with COPD, over several years, we found that AECOPD was associated with increased 30-day and 1-year incidences of acute cardiovascular event (AMI, HF, AF, $\mathrm{PE}$, ischemic stroke, and hemorrhagic stroke). The observed associations were robust across different analytic assumptions. Our findings should encourage researchers to elucidate the mechanisms linking AECOPD to the development of acute cardiovascular events. For clinicians, our data support an importance of AECOPD as an opportunity to implement cardiovascular prevention measures in this high-risk population.

Corresponding Author: Tadahiro Goto, MD, MPH; Department of Emergency Medicine Massachusetts General Hospital, Boston, MA, USA (e-mail: tag695@mail.harvard.edu).

Details of Contributors and the Name of the Guarantor T.G. takes responsibility for the paper as a whole. T.G., Y.J.S., M.K.F., C.A.C., and K.H. conceived the study. C.A.C. obtained research funding and supervised the conduct of the study. T.G., Y.J.S., M.K.F., and K.H. provided statistical advice. T.G. and M.K.F. analyzed the data. T.G. drafted the manuscript, and all authors contributed substantially to its revision. The content is solely the responsibility of the authors and does not necessarily represent the official views of the Agency for Healthcare Research and Quality.
Financial Support This study was supported by the grant RO1 HS023305 (Camargo) from the Agency for Healthcare Research and Quality (Rockville, MD). Dr. Goto was supported by grants from St Luke's Life Science Institute and Uehara Kinen Memorial Foundation (Tokyo, Japan).

\section{Compliance with Ethical Standards:}

The institutional review board of Massachusetts General Hospital approved this study.

Conflict of Interest: The authors declare that they do not have a conflict of interest.

\section{REFERENCES}

1. Chronic Respiratory Diseases. World Health Organization. 2000; http:// www.who.int/gard/publications/chronic_respiratory_diseases.pdf. Accessed May 1, 2018.

2. Adeloye D, Chua S, Lee C, et al. Global and regional estimates of COPD prevalence: systematic review and meta-analysis. $J$ Glob Health. 2015;5(2):020415.

3. Aleva FE, Voets LW, Simons So, de Mast $\mathbf{Q}$, van der Ven AJ, Heijdra YF. Prevalence and localization of pulmonary embolism in unexplained acute exacerbations of COPD: a systematic review and meta-analysis. Chest. 2016; 151(3):544-554.

4. Chen W, Thomas J, Sadatsafavi M, FitzGerald JM. Risk of cardiovascular comorbidity in patients with chronic obstructive pulmonary disease: a systematic review and meta-analysis. Lancet Respir Med. 2015;3(8):631-639.

5. Calverley PM, Anderson JA, Celli B, et al. Salmeterol and fluticasone propionate and survival in chronic obstructive pulmonary disease. $N$ Engl J Med. 2007;356(8):775-789.

6. McGarvey LP, John M, Anderson JA, Zvarich M, Wise RA, Committee TCE. Ascertainment of cause-specific mortality in COPD: operations of the TORCH Clinical Endpoint Committee. Thorax. 2007;62(5):411-415.

7. Rizkallah J, Man SF, Sin DD. Prevalence of pulmonary embolism in acute exacerbations of COPD: a systematic review and metaanalysis. Chest. 2009;135(3):786-793.

8. Celli BR, Decramer M, Wedzicha JA, et al. An Official American Thoracic Society/European Respiratory Society Statement: research questions in chronic obstructive pulmonary disease. Am J Respir Crit Care Med. 2015;191(7):e4-e27.

9. Soler-Cataluna JJ, Martinez-Garcia MA, Roman Sanchez P, Salcedo E, Navarro M, Ochando R. Severe acute exacerbations and mortality in patients with chronic obstructive pulmonary disease. Thorax. 2005;60(11):925-931.

10. Ford ES. Hospital discharges, readmissions, and ED visits for COPD or bronchiectasis among US adults: findings from the nationwide inpatient sample 2001-2012 and Nationwide Emergency Department Sample 2006-2011. Chest. 2015;147(4):989-998.

11. Donaldson GC, Hurst JR, Smith CJ, Hubbard RB, Wedzicha JA. Increased risk of myocardial infarction and stroke following exacerbation of COPD. Chest. 2010;137(5):1091-1097.

12. Portegies ML, Lahousse L, Joos GF, et al. Chronic obstructive pulmonary disease and the risk of stroke. The Rotterdam Study. Am J Respir Crit Care Med. 2016;193(3):251-258.

13. Seemungal T, Harper-Owen R, Bhowmik A, et al. Respiratory viruses, symptoms, and inflammatory markers in acute exacerbations and stable chronic obstructive pulmonary disease. Am J Respir Crit Care Med. 2001;164(9):1618-1623.

14. Smeeth L, Thomas SL, Hall AJ, Hubbard R, Farrington $\mathbf{P}$, Vallance $\mathbf{P}$. Risk of myocardial infarction and stroke after acute infection or vaccination. $N$ Engl J Med. 2004;351(25):2611-2618.

15. Falk JA, Kadiev S, Criner GJ, Scharf SM, Minai OA, Diaz P. Cardiac disease in chronic obstructive pulmonary disease. Proc Am Thorac Soc. 2008;5(4):543-548.

16. Hasegawa $\mathbf{K}$, Tsugawa $\mathbf{Y}$, Chang $\mathbf{Y}$, Camargo CA, Jr. Risk of an asthma exacerbation after bariatric surgery in adults. J Allergy Clin Immunol. 2015;136(2):288-294 e288.

17. Whitaker HJ, Farrington CP, Spiessens B, Musonda P. Tutorial in biostatistics: the self-controlled case series method. Stat Med. 2006;25(10):1768-1797.

18. Overview of the State Inpatient Databases (SID). Healthcare Cost and Utilization Project. Agency for Healthcare Research and 
Quality. http://www.hcup-us.ahrq.gov/sidoverview.jsp. Accessed May 1, 2018.

19. Hasegawa K, Camargo CA, Jr. Prevalence of blood eosinophilia in hospitalized patients with acute exacerbation of COPD. Respirology. 2016;21(4):761-764

20. Yale New Haven Health Services Corporation/Center for Outcomes Research \& Evaluation. 2014 Measures Updates and Specifications Report Hospital-Level 30-Day Risk-Standardized Readmission Measures. 2014; http://altarum.org/sites/default/files/uploaded-publicationfiles/Rdmsn_Msr_Updts_HWR_0714_0.pdf. Accessed May 1, 2018.

21. Ginde AA, Tsai CL, Blanc PG, Camargo CA, Jr. Positive predictive value of ICD-9-CM codes to detect acute exacerbation of COPD in the emergency department. Jt Comm J Qual Patient Saf. 2008;34(11):678-680.

22. Stein BD, Bautista A, Schumock GT, et al. The validity of International Classification of Diseases, Ninth Revision, Clinical Modification diagnosis codes for identifying patients hospitalized for COPD exacerbations. Chest. 2012;141(1):87-93.

23. Kiyota Y, Schneeweiss S, Glynn RJ, Cannuscio CC, Avorn J, Solomon DH. Accuracy of Medicare claims-based diagnosis of acute myocardial infarction: estimating positive predictive value on the basis of review of hospital records. Am Heart J. 2004;148(1):99-104.

24. Chen J, Normand SL, Wang Y, Krumholz HM. National and regional trends in heart failure hospitalization and mortality rates for Medicare beneficiaries, 1998-2008. JAMA. 2011;306(15):1669-1678.

25. Chen J, Hsieh AF, Dharmarajan K, Masoudi FA, Krumholz HM. National trends in heart failure hospitalization after acute myocardial infarction for Medicare beneficiaries: 1998-2010. Circulation. 2013;128(24):2577-2584.

26. Patel NJ, Deshmukh A, Pant S, et al. Contemporary trends of hospitalization for atrial fibrillation in the United States, 2000 through 2010: implications for healthcare planning. Circulation. 2014;129(23):23712379 .

27. Fang MC, Fan D, Sung SH, et al. Outcomes in adults with acute pulmonary embolism who are discharged from emergency departments: the Cardiovascular Research Network Venous Thromboembolism study. JAMA Intern Med. 2015;175(6): 1060-1062.

28. Tirschwell DL, Longstreth WT, Jr. Validating administrative data in stroke research. Stroke. 2002;33(10):2465-2470.

29. Birman-Deych E, Waterman AD, Yan Y, Nilasena DS, Radford MJ, Gage BF. Accuracy of ICD-9-CM codes for identifying cardiovascular and stroke risk factors. Med Care. 2005;43(5):480-485.

30. Olson KL, Wood MD, Delate T, et al. Positive predictive values of ICD-9 codes to identify patients with stroke or TIA. Am $J$ Manag Care. 2014;20(2):e27-34.
31. Sharif R, Parekh TM, Pierson KS, Kuo YF, Sharma G. Predictors of early readmission among patients 40 to 64 years of age hospitalized for chronic obstructive pulmonary disease. Ann Am Thorac Soc. 2014;11(5):685-694.

32. Gunen H, Gulbas G, In E, Yetkin O, Hacievliyagil SS. Venous thromboemboli and exacerbations of COPD. Eur Respir J. 2010;35(6):1243-1248.

33. Terzano C, Romani S, Conti V, Paone G, Oriolo F, Vitarelli A. Atrial fibrillation in the acute, hypercapnic exacerbations of COPD, Eur Rev Med Pharmacol Sci. 2014;18(19):2908-2917.

34. Morgan AD, Herrett E, De Stavola BL, Smeeth L, Quint JK. COPD disease severity and the risk of venous thromboembolic events: a matched case-control study. Int $J$ Chron Obstruct Pulmon Dis. 2016;11:899-908.

35. Sinden NJ, Stockley RA. Systemic inflammation and comorbidity in COPD: a result of 'overspill' of inflammatory mediators from the lungs? Review of the evidence. Thorax. 2010;65(10):930-936.

36. Sin DD, Anthonisen NR, Soriano JB, Agusti AG. Mortality in COPD: role of comorbidities. Eur Respir J. 2006;28(6):1245-1257.

37. Patel AR, Kowlessar BS, Donaldson GC, et al. Cardiovascular risk, myocardial injury, and exacerbations of chronic obstructive pulmonary disease. Am J Respir Crit Care Med. 2013;188(9): 1091-1099.

38. Clarenbach CF, Senn O, Sievi NA, et al. Determinants of endothelial function in patients with COPD. Eur Respir J. 2013;42(5):1194-1204.

39. Rutten FH, Zuithoff NP, Hak E, Grobbee DE, Hoes AW. Beta-blockers may reduce mortality and risk of exacerbations in patients with chronic obstructive pulmonary disease. Arch Intern Med. 2010;170(10):880-887.

40. Ekstrom MP, Hermansson AB, Strom KE. Effects of cardiovascular drugs on mortality in severe chronic obstructive pulmonary disease. Am J Respir Crit Care Med. 2013;187(7):715-720.

41. Salpeter SR, Ormiston TM, Salpeter EE. Cardiovascular effects of betaagonists in patients with asthma and COPD: a meta-analysis. Chest. 2004; 125(6):2309-2321.

42. Aaron CP, Schwartz JE, Hoffman EA, et al. A longitudinal cohort study of aspirin use and progression of emphysema-like lung characteristics on CT imaging: the MESA Lung Study. Chest. 2017.

43. Goto T, Faridi MK, Camargo CA, Hasegawa $\mathbf{K}$. The association of aspirin use with severity of acute exacerbation of chronic obstructive pulmonary disease: a retrospective cohort study. NPJ Prim Care Respir Med. 2018;28(1):7.

44. Hasegawa K, Tsugawa Y, Tsai CL, Brown DF, Camargo CA, Jr. Frequent utilization of the emergency department for acute exacerbation of chronic obstructive pulmonary disease. Respir Res. 2014;15:40. 